\title{
Dynamical Adjustment of the Northern Hemisphere Surface Air Temperature Field: Methodology and Application to Observations*
}

\author{
Brian V. SMoliak, ${ }^{+}$John M. Wallace, Pu Lin, ${ }^{\#}$ AND Qiang Fu \\ Department of Atmospheric Sciences, University of Washington, Seattle, Washington
}

(Manuscript received 5 February 2014, in final form 10 November 2014)

\begin{abstract}
The influence of atmospheric circulation changes reflected in spontaneously occurring sea level pressure (SLP) anomalies upon surface air temperature (SAT) variability and trends is investigated using partial least squares (PLS) regression, a statistical method that seeks to maximally explain covariance between a predictand time series or field and a predictor field. Applying PLS regression in any one of the three variants described in this study (pointwise, PC-wise, and fieldwise), the method yields a dynamical adjustment to the observed NH SAT field that accounts for approximately $50 \%$ of the variance in monthly mean, cold season data. It is shown that PLS regression provides a more parsimonious and statistically robust dynamical adjustment than an adjustment method based on the leading principal components of the extratropical SLP field. The usefulness of dynamical adjustment is demonstrated by applying it to the attribution of cold season SAT trends in two reference intervals: 1965-2000 and 1920-2011. The adjustment is shown to reconcile much of the spatial structure and seasonal differences in the observed SAT trends. The dynamically adjusted SAT fields obtained from this analysis provide datasets capable of being analyzed for residual variability and trends associated with thermodynamic and radiative processes.
\end{abstract}

\section{Introduction}

On local and regional scales, the month-to-month and year-to-year variability of surface air temperature (SAT) is strongly influenced by warming and cooling associated with changing atmospheric circulation patterns. This dynamically induced variability is particularly strong over land poleward of $30^{\circ} \mathrm{N}$ during the boreal cold season, where the cold continental interiors are ventilated to varying degrees by relatively mild maritime air masses. Because of this "dynamical noise" in the time-varying SAT field, it is only when temperatures are averaged over

\footnotetext{
* Supplemental information related to this paper is available at the Journals Online website: http://dx.doi.org/10.1175/JCLI-D-1400111.s1.

${ }^{+}$Current affiliation: The Climate Corporation, Seattle, Washington.

\# Current affiliation: Atmospheric and Oceanic Sciences Program, Princeton University/NOAA/Geophysical Fluid Dynamics Laboratory, Princeton, New Jersey.
}

Corresponding author address: Brian V. Smoliak, The Climate Corporation, 419 Occidental Ave. S., Suite 201, Seattle, WA 98104. E-mail: bsmoliak@climate.com large areas and smoothed in time that the signature of human-induced global warming becomes clearly evident (Bradley et al. 1987; Wallace 1995).

Early studies of atmospheric teleconnections by Walker and Bliss $(1932,1937)$ established that SAT and SLP anomalies exhibit concurrent relationships across large spatial scales, evident in the manner with which they used linear combinations of SAT and SLP anomalies to define teleconnection indices. Simultaneous relationships between surface temperature and circulation fields were later formalized using multiple regression equations and leveraged for the purposes of prediction (Klein et al. 1959) and specification (Klein 1962) of local surface temperatures from 700-hPa geopotential heights. Following these earlier studies, Klein (1983) developed a set of specification equations based on a pointwise screening technique, which identified a limited set of gridpoint $700-\mathrm{hPa}$ geopotential height observations that best explain the variance of monthly-mean SAT during winter. Klein and Walsh (1983) subsequently compared the pointwise screening approach to an alternative using empirical orthogonal functions (EOFs). Klein and Walsh used a variety of skill metrics and test cases to demonstrate that pointwise screening is more effective than EOF analysis for specifying local temperature on the 
basis of 700-hPa height patterns. Van den Dool et al. (1993) extended Klein's pointwise screening technique to examine the consistency of interdecadal trends in 700-hPa geopotential height and local SAT. They did so by analyzing residuals associated with a specification of monthly-mean SAT at a variety of sites throughout the United States, given the observed extratropical $\mathrm{NH}$ 700 -hPa geopotential height field between $180^{\circ}$ and $50^{\circ} \mathrm{W}$.

Wallace et al. (1995) used regression analysis to identify the spatial pattern in the NH SAT departure field (i.e., the SAT anomaly at each land grid point minus the SAT anomaly averaged over all land regions poleward of $20^{\circ} \mathrm{N}$ ) that occurs in linear combination with month-to-month variations in the $\mathrm{NH}$-mean SAT time series (NHSAT). The time-varying index of this socalled cold ocean-warm land (COWL) pattern accounts for nearly half of the month-to-month variance of NHSAT. Subtracting out the component of the NHSAT time series variability that is linearly congruent with the COWL index yields a residual NHSAT time series that is visibly smoother and exhibits a substantially reduced late twentieth-century boreal cold season warming trend (Wallace et al. 1995, 1996).

Climate models predict that SAT over the continents should rise more rapidly than sea surface temperature (SST) in response to the buildup of greenhouse gases (Broccoli et al. 1998; Jain et al. 1999; Braganza et al. 2003; Sutton et al. 2007; Meehl et al. 2007a; Joshi et al. 2008). Hence, the observed trend toward the positive polarity of the COWL pattern is not entirely dynamically induced; at least part of it should be viewed as an integral part of the signature of the human-induced global warming (Broccoli et al. 1998). Mindful of this inherent ambiguity in a dynamical index based on SAT, other investigators have sought to define the dynamical contribution to the hemispheric-mean SAT trend on the basis of the sea level pressure (SLP) field. Hurrell (1996) used indices of the North Atlantic Oscillation (NAO) and El Niño-Southern Oscillation (ENSO) as a basis for defining a dynamically adjusted global-mean SAT (GSAT). Thompson et al. (2000) and Quadrelli and Wallace (2004) used principal component (PC)-based indices of the northern annular mode (NAM) and the Pacific-North American (PNA) pattern. An alternative approach is to regress the SLP field onto a detrended or high-pass-filtered time series of NHSAT. The resulting spatial pattern can be used to define an SLP index that represents the dynamically induced variability, for example, in a reduced phase space defined by the leading patterns of an EOF expansion of the time-varying SLP field (Thompson et al. 2009). Fyfe et al. (2010) applied the same method as Thompson et al. (2009) to a multimodel ensemble of twentieth-century simulations from phase 3 of the Coupled Model Intercomparison Project (CMIP3; Meehl et al. 2007b). Wallace et al. (2012) used an approach similar to Thompson et al. (2009), but employed partial least squares (PLS) regression for the dynamical adjustment and focused their analysis on dynamical contribution to the observed late twentiethcentury boreal cold season warming poleward of $40^{\circ} \mathrm{N}$.

In this paper, we extend previous applications of PLS regression to detect and remove dynamically induced variability in climate time series. Following Wallace et al. (2012), we seek the best linear prediction of SAT by forming statistical predictors from anomaly patterns in the SLP field that are tailored to maximally explain concurrent SAT variations. Rather than focusing exclusively on globally or hemispherically averaged SAT time series, we also consider the possibility of adjusting the entire NH SAT field poleward of $20^{\circ} \mathrm{N}$, which can be used for attribution of regional or local climate change or for homogenizing the individual runs in an ensemble of climate model integrations. We use the SLP field to derive monthly dynamical adjustments for both large area-average time series and for individual SAT gridpoint time series, and we link the two using an algebraic identity in the appendix. To judge the effectiveness of the PLS regression-based dynamical adjustment, we will compare it with another linear adjustment based on principal component regression (PCR), identical to one of the approaches used by Thompson et al. (2009) to estimate dynamically induced variability in global-mean surface temperature (GST) and GSAT.

Three variants of dynamical adjustment based on PLS regression will be compared. When used in a "pointwise" manner, the method is applied to a single SAT time series, either in an area-average sense, in which only the area-average time series is adjusted, or in a disaggregated sense, in which each grid point in the SAT field is adjusted independently. When used in a "PC-wise" manner, the method is applied independently to the leading principal components of the SAT field poleward of $20^{\circ} \mathrm{N}$, and when used in a "fieldwise" manner, the method is applied to the entire SAT field at once.

As a basis for evaluating the different methodologies, we compare the fraction of the variance of monthlymean SAT data accounted for by the dynamical adjustment at each SAT grid point and the fraction of the SAT variance explained averaged over all $\mathrm{NH}$ land grid points poleward of $20^{\circ} \mathrm{N}$. We focus on the extratropical NH during the boreal cold season (November-April), in which the dynamical contribution to the SAT variability is largest and prior studies offer guidance as to the patterns that have the strongest impact on SAT.

This article is organized as follows. Section 2 describes the datasets used in the analyses performed for this 
study. Section 3 describes PLS regression (PLSR), relates PLSR to more established methodologies, distinguishes between the three aforementioned PLSR variants, and systematically compares the approaches to each other and to PCR, which is a baseline from previous studies. Section 4 illustrates the effectiveness of dynamical adjustment with a pair of applications in which dynamical contributions to SAT trends are diagnosed. We consider the observed SAT trends during the reference interval 1965-2000, and the observed SAT trends during the more extended reference interval 1920-2011. The paper concludes with a discussion and summary section.

\section{Data}

In this study we make use of the National Oceanic and Atmospheric Administration (NOAA) Merged LandOcean Surface Temperature (MLOST) analysis, version 3.5.3 (Vose et al. 2012), with its land component, the Global Historical Climatology Network (GHCN), version 3 (Lawrimore et al. 2011), and ocean component, the Extended Reconstructed Sea Surface Temperature (ERSST), version 3b (Smith et al. 2008). The SAT data are obtained as monthly-mean anomaly fields on a regular $5^{\circ}$ latitude by $5^{\circ}$ longitude global grid. Unless otherwise noted, maps are based on the MLOST dataset, which includes some reconstruction to fill in missing data. Time series and statistics in tables are based on the land-only GHCN dataset, which is more observationally pure as it is not produced with filling in of missing data. Use of the MLOST dataset facilitates a clearer picture of spatial patterns, and use of the land-only GHCN dataset eliminates the chance that SST variability may affect our analysis of coastal grid cells.

We use the SLP field to investigate the role of the atmospheric circulation in generating SAT variability. We chose SLP rather than lower-tropospheric geopotential height (e.g., $700 \mathrm{hPa}$ ) because the latter is arguably more influenced by the SAT field, both in terms of the variability and trends. In the lower troposphere, the thickness between two isobaric levels is a proxy for the mean temperature of the underlying air mass, whereas the SLP responds to the entire column above it and is less influenced by near-surface conditions. Thus, the asymmetric relationship between predictors and predictand(s) assumed with PLS regression is better justified with the SLP field.

The observational SLP field used in this study is from the NOAA-CIRES Twentieth-Century Reanalysis (20CR), version 2 (Compo et al. 2011). The SLP data are obtained as monthly mean fields on a regular $2^{\circ}$ latitude by $2^{\circ}$ longitude global grid. We have used this product partly because it is the only state-of-the-art SLP dataset that extends back to the early twentieth century. We also justify the use of the 20CR out of concern that the spurious discontinuities in other reanalysis products that coincide with changes in the observing system could contaminate the time series of the adjustment.

Monthly SAT and SLP anomaly fields are analyzed for the time period from November 1919 through October 2011. Monthly anomalies are calculated with respect to the long-term calendar month mean. Our boreal cold season (November-April) extends across the divide between calendar years. Following the same convention used in dealing with the conventional winter season (December-February), we assign the cold season extending from year $n$ to year $n+1$ to year $n+1$ (e.g., from November 1964-April 1965 to the calendar year 1965). Our boreal warm season is defined as May-October.

\section{Dynamical adjustment with partial least squares regression}

PLS regression is a matrix method of analysis that seeks to specify one or more dependent variables $\mathbf{Y}$ which we will refer to as the predictand(s), with a set of independent variables $\mathbf{X}$ which we will refer to as predictors. PLS regression specifies a spatiotemporal predictand field $y\left(s_{y}, t\right)$ by a predictor field $x\left(s_{x}, t\right)$ with identical sampling in the time dimension $t$ but possibly different sampling in the space dimension $\left(s_{y} \neq s_{x}\right)$ :

$$
\hat{\mathbf{Y}}=\mathbf{Z B C}^{\mathrm{T}} \text {, }
$$

where $\mathbf{Z}$ are PLS predictor covariates, $\mathbf{B}$ is a diagonal matrix containing regression coefficients as its diagonal elements, $\mathbf{C}$ is the weight matrix corresponding to the dependent variable $\mathbf{Y}$, and $\mathrm{T}$ is the transpose operator. The specification equations are developed by decomposing $\mathbf{X}$ and $\mathbf{Y}$ as the product of a common set of mutually orthogonal components in the sampling dimension, called "latent vectors" or "PLS predictors", and specific sets of loadings. The independent variables are decomposed according to the relation:

$$
\mathbf{X}=\mathbf{Z} \mathbf{P}^{\mathrm{T}} \quad \text { and } \quad \mathbf{Z}^{\mathrm{T}} \mathbf{Z}=\mathbf{I},
$$

where $\mathbf{Z}$ are the PLS predictors, $\mathbf{P}$ are its loadings, and $\mathbf{I}$ is the identity matrix. As discussed by Abdi (2010), any set of mutually orthogonal vectors that span the space of $\mathbf{X}$ could be used for $\mathbf{Z}$. For example, the principal components (PCs) of $\mathbf{X}$ could be used to specify $\mathbf{Y}$. This procedure is known as $\mathrm{PC}$ regression (PCR). However, with PCR there is no guarantee that the structures that best explain the variance of the predictor field are relevant to the predictand(s). The goal of PLS regression is 
to find structures in the predictor field that best explain the variance of the predictand field. Thus, PLS predictors are tailored by finding two sets of weights, $\mathbf{w}$ and c, which are used to form linear combinations of the independent and dependent variables with maximum covariance:

$$
\mathbf{z}=\mathbf{X} \mathbf{w} \quad \text { and } \quad \mathbf{u}=\mathbf{Y} \mathbf{c}
$$

subject to the constraints that

$$
\mathbf{w}^{\mathrm{T}} \mathbf{w}=1, \quad \mathbf{z}^{\mathrm{T}} \mathbf{z}=1, \quad \text { and } \quad \mathbf{z}^{\mathrm{T}} \mathbf{u}=\max .
$$

There is no method for determining the entire set of PLS predictors in a single operation. PLS predictors are determined iteratively through a process of finding weights, forming a single PLS predictor as a linear combination of $\mathbf{X}$, and subtracting the PLS predictor from $\mathbf{X}$ and $\mathbf{Y}$ by ordinary least squares regression to form residual matrices. The next PLS predictor is formed from these partially deflated matrices. PLS regression derives its "partial" nomenclature from this property, which ensures that the PLS predictors are mutually orthogonal and represent nonoverlapping portions of the predictor variance.

The practice of determining weights depends on the number of predictands. In the case of a multivariate predictand, the specification is described by (1), $\mathbf{w}$ and $\mathbf{c}$ are determined as the first right and left singular vectors, respectively, of $\mathbf{X}^{\mathrm{T}} \mathbf{Y}$. By extention, the linear combinations $\mathbf{z}$ and $\mathbf{u}$ are the first eigenvectors of $\mathbf{X} \mathbf{X}^{\mathrm{T}} \mathbf{Y} \mathbf{Y}^{\mathrm{T}}$ and $\mathbf{Y} \mathbf{Y}^{\mathrm{T}} \mathbf{X} \mathbf{X}^{\mathrm{T}}$, respectively.

The process of determining $\mathbf{w}, \mathbf{c}, \mathbf{z}$, and $\mathbf{u}$ is identical to the first step of singular value decomposition (SVD) analysis (Bretherton et al. 1992; Wallace et al. 1992), which has subsequently come to be referred to as maximum covariance analysis (MCA). Unlike MCA, which yields nonorthogonal expansion coefficient time series that maximize squared covariance between the $\mathbf{X}$ and $\mathbf{Y}$ spatial patterns via a single SVD operation on their cross-covariance matrix, PLS regression yields mutually orthogonal expansion coefficient time series that maximize covariance between themselves and the dependent variables in (4) via an iterative process. Another important distinction between the two methods is that in MCA $\mathbf{X}$ and $\mathbf{Y}$ are interchangeable, whereas in PLS regression $\mathbf{X}$ is used to predict of $\mathbf{Y}$. PLS regression also bears some similarity to canonical correlation analysis (CCA), which seeks maximally correlated linear combinations of two input fields. Like PLS regression, CCA yields mutually orthogonal singular vector time series that may be used to specify Y; however, unlike PLS regression, CCA does not distinguish between predictor and predictand fields. Another distinction is that CCA is typically performed in an optimally truncated PC space (Barnett and Preisendorfer 1987), whereas PLS regression, like MCA, is performed upon the full rank $\mathbf{X}$ and $\mathbf{Y}$ matrices and is therefore much more straightforward to apply and less susceptible to "overfitting" (Davis 1976). The goal of this study is to introduce and compare variants of PLS regression; an exhaustive quantitative comparison of PLSR to other matrix methods of analysis is beyond its scope.

In the case of a univariate predictand, the PLS regression specification equation in (1) reduces to

$$
\hat{\mathbf{y}}=\mathbf{Z B} \text {, }
$$

and $\mathbf{w}$ is determined by regressing $\mathbf{X}$ onto $\mathbf{y}$ :

$$
\mathbf{w}=\mathbf{X y} \text {. }
$$

When the input variables are column centered and standardized to a variance of unity, as is common in applications of PLSR (Abdi 2010), the weights are crosscorrelation coefficients, and the decomposition of $\mathbf{X}$ in (2) yields PLS predictors that are maximally correlated with the predictand.

In summary, the primary product of PLS regression is a set of PLS predictor time series that express the timevarying amplitude of structures in the predictor field that are maximally correlated with the predictand(s). Using linear combinations of gridpoint time series in the predictor field (i.e., patterns), as opposed to time series for individual grid points as in the pointwise screening method employed in previous SAT specification studies (Klein 1983; Van den Dool et al. 1993), yields a more parsimonious specification. In an application of dynamical adjustment to the NH SAT field, Smoliak (2013) found that the pointwise screening method generally requires more covariates to explain as much of the predictand variance as PLS regression does and is slightly less robust under cross validation.

A noteworthy by-product of PLS regression is the independent variable loading matrix $\mathbf{P}$ obtained by regressing $\mathbf{X}$ onto the PLS predictors $\mathbf{Z}$. Unlike the PLS predictor time series, the loading patterns in $\mathbf{P}$ are not mutually orthogonal; however, they may be used to interpret the dynamical relationship between predictor and predictand.

Examples showing how PLS regression can be applied to climate data can be found in the literature, for example, in paleoclimate reconstruction (Kalela-Brundin 1999), seasonal prediction (McIntosh et al. 2005), and diagnosing dynamically induced variability in climate time series (Smoliak et al. 2010; Wallace et al. 2012). Whereas Smoliak et al. (2010) and Wallace et al. (2012) 
limited their analyses to area-average time series, here we apply PLS regression to the entire NH SAT field. In the following subsections, we briefly describe each of the three variants of PLS regression and direct the reader to the appendix for a more detailed description of methodological considerations.

\section{a. Pointwise dynamical adjustment}

In its simplest form, PLS regression is applied in a pointwise manner (i.e., to an individual target time series, here either a gridpoint or an area-averaged temperature time series). The target time series is high-pass filtered to prevent fitting trends in the two fields (see the appendix for details), and then correlated with the SLP predictors to form a one-point cross-correlation map that serves as the weighting function (w). Next the monthly, standardized, predictor SLP field $(\mathbf{X})$, weighted by the cosine of latitude, is projected onto the cross-correlation pattern in (3) to obtain a time-varying index of the pattern (i.e., a PLS predictor $\mathbf{z}$ ). The use of standardized predictor data ensures that the predictors are weighted by in accordance with their correlation with the predictand(s); this prevents predictors with large variances and low correlations from unduly influencing the linear combination.

Once a PLS predictor time series is determined, it is regressed out of the predictor field and the "unfiltered" predictand. This step creates a set of residual variables that are the basis for determining the next PLS predictor. To prevent overfitting, only a limited set of PLS predictors are retained for the purposes of specification, as determined through cross validation. The cross validation of the dynamical adjustment based on pointwise PLS regression is cumbersome because the optimal number of SLP-based predictors to retain varies from grid point to grid point. In lieu of cross validation, we elected to use only the leading three SLP patterns at each grid point (see the appendix for details). The simplification of the method comes at the expense of leaving "unexplained" a small fraction of the dynamically induced SAT variance that might have been explained by subsequent passes. We will return to this point in the discussion and summary section.

By way of example, we apply PLS regression to the time series of monthly-mean, cold season SAT averaged over land areas poleward of $20^{\circ} \mathrm{N}$ (NHSAT). The cold season-mean NHSAT anomaly time series is shown in Fig. 1a, followed by the dynamically induced component of the variability estimated using three PLS predictors in Fig. 1b, and the residual NHSAT time series in Fig. 1c. Dynamical adjustment with PLS regression accounts for much of the month-to-month and year-to-year

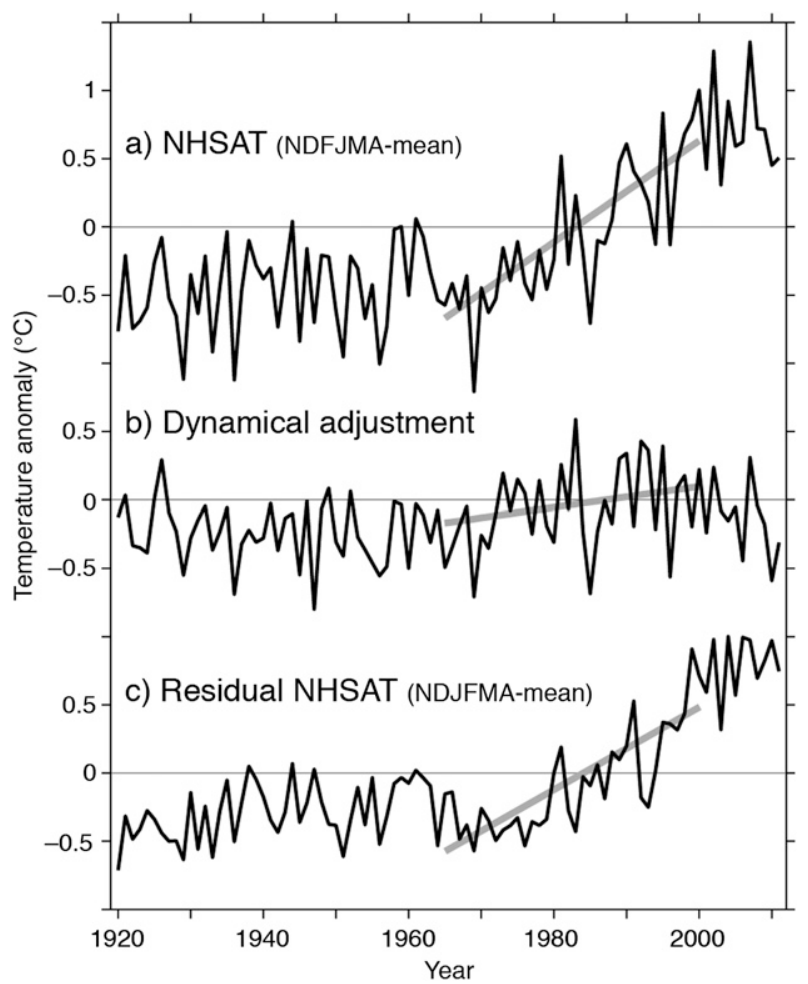

FIG. 1. (a) Boreal cold season-mean NHSAT anomaly time series, 1920-2011, based on the land-only GHCN dataset, (b) the SLP-based dynamical adjustment to (a) using three passes of PLS regression as described in the text, and (c) the residual cold seasonmean NHSAT anomaly time series. Linear trends 1965-2000, an interval marked by rapid warming over the Northern Hemisphere continents poleward of $40^{\circ} \mathrm{N}$, are shown in light gray. Anomalies are displayed with respect to the base period 1971-2000.

variability of NHSAT, as evidenced by the contrast between the highly variable dynamical component and smoother residual time series. Dynamical adjustment can also be used to estimate the dynamical contributions to SAT trends. For example, the large positive linear trend evident in the unadjusted NHSAT time series over the period 1965-2000 (Fig. 1a, gray line) is reduced after applying dynamical adjustment (Fig. 1b), leaving a residual trend that may be interpreted as primarily radiatively or thermodynamically induced (Fig. 1c).

As a second example, we apply PLS regression to the time series of monthly-mean cold season SAT at reference grid points in the interior of Eurasia and North America, where the dynamical contribution to the temperature variability and SAT trends $1965-2000$ is particularly strong. When applying PLS regression to local time series, we explored varying the size of the SLP predictor field from the entire $\mathrm{NH}$ poleward of $20^{\circ} \mathrm{N}$ down to a small region centered on the grid point. We found that the results were not very sensitive to the areal extent of the predictor field within the range of $30^{\circ}$ to $180^{\circ}$ of 


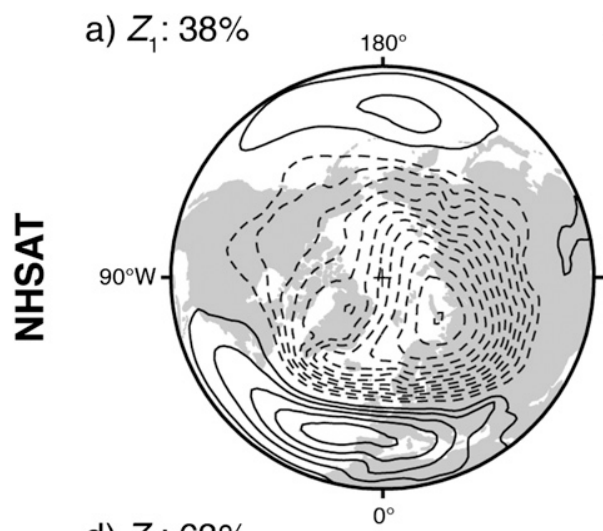

d) $Z_{1}: 63 \%$

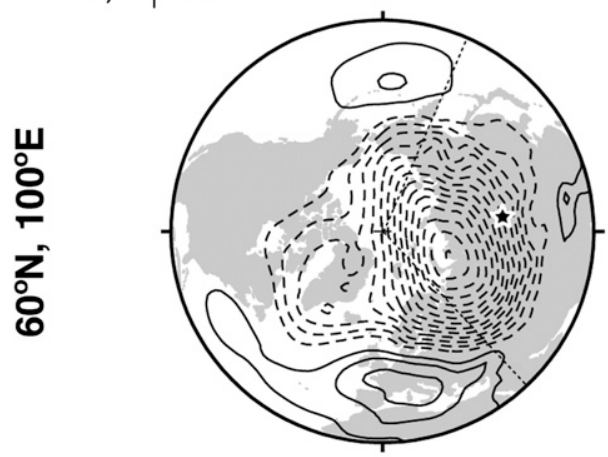

g) $Z_{1}: 54 \%$

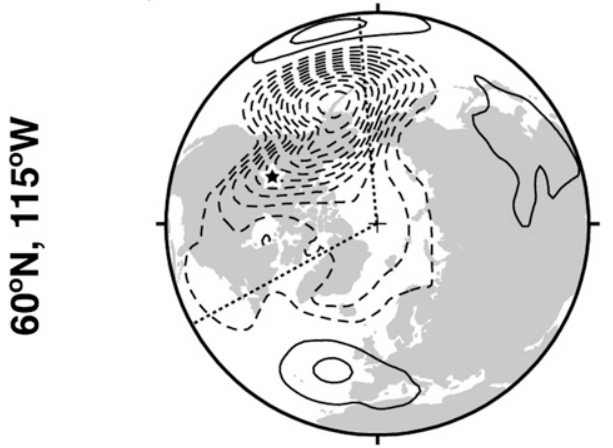

b) $Z_{2}: 6 \%$

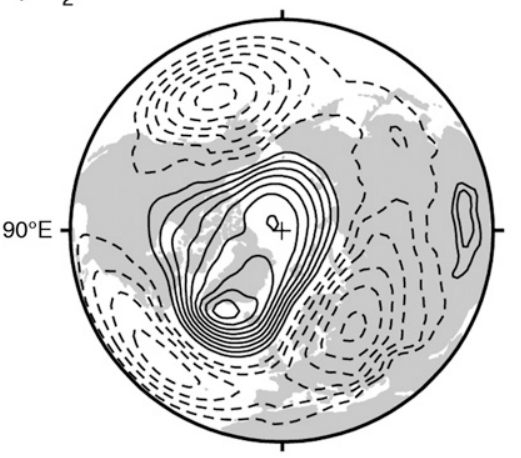

e) $Z_{2}: 3 \%$

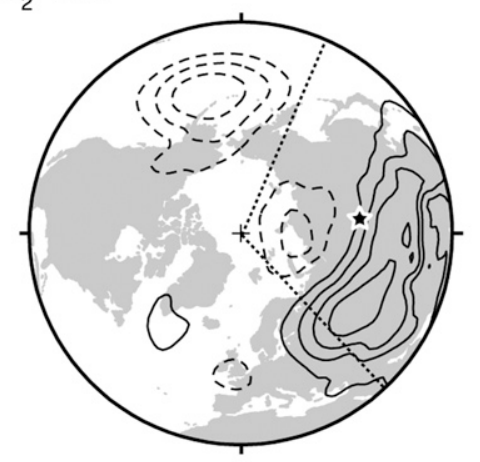

h) $Z_{2}: 11 \%$

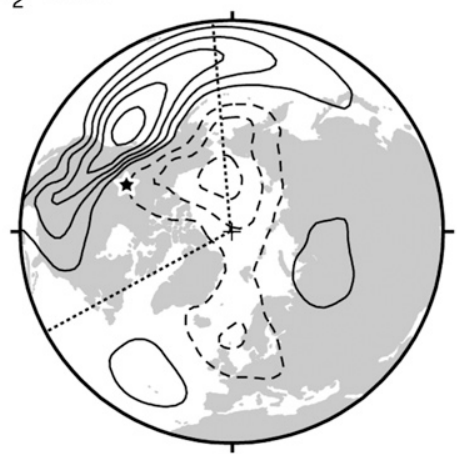

c) $Z_{3}: 4 \%$

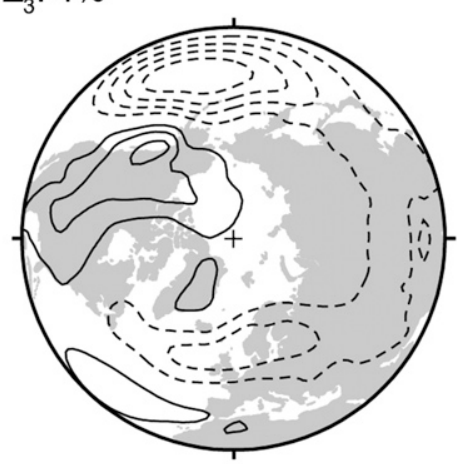

f) $Z_{3}: 3 \%$

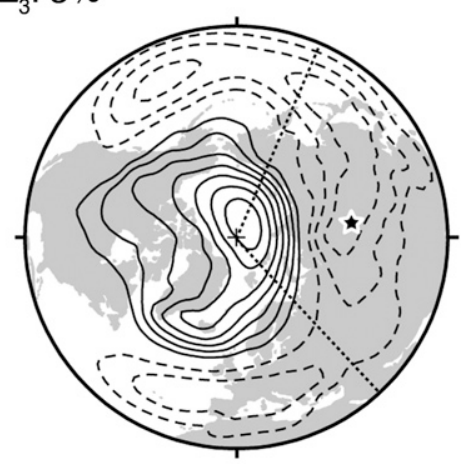

i) $Z_{3}: 3 \%$

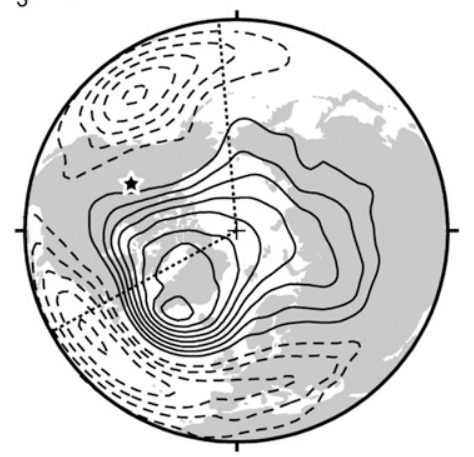

FIG. 2. SLP regression maps associated with the leading three PLS predictors of (a)-(c) the NHSAT time series, using a hemispheric predictor field; as well as (d)-(f) SAT time series at a Eurasian grid point $\left(60^{\circ} \mathrm{N}, 100^{\circ} \mathrm{E}\right)$; and (g)-(i) SAT at a North American grid point $\left(60^{\circ} \mathrm{N}, 115^{\circ} \mathrm{W}\right)$ using $120^{\circ}$ longitudinal predictor fields centered on the grid point. The maps are regressions of the monthly-mean NH cold season SLP field onto the respective standardized PLS predictors. The areal extent of the predictor field is outlined with dotted black lines, beyond which the SLP regression is shown for the purposes of illustration. The contour interval is $0.5 \mathrm{hPa}\left(\mathrm{std}\right.$ dev) ${ }^{-1}$ of the corresponding PLS predictor, negative values are dashed, and the zero line is omitted. The fraction of predictand SAT variance explained by the corresponding PLS predictor is shown above left of each map.

longitude (see the appendix for details). Our adjustment of the NH SAT field is based on SLP predictor fields that extend from $20^{\circ}$ to $90^{\circ} \mathrm{N}$ and across $120^{\circ}$ longitudinal sectors centered on the reference grid point. Figure 2 shows SLP regression maps associated with the leading three PLS predictors of NHSAT (Figs. 2a-c), SAT at a Eurasian grid point $\left(60^{\circ} \mathrm{N}, 100^{\circ} \mathrm{E}\right.$; Figs. $\left.2 \mathrm{~d}-\mathrm{f}\right)$, and SAT at a North American grid point $\left(60^{\circ} \mathrm{N}, 115^{\circ} \mathrm{W}\right.$; Figs. $\left.2 \mathrm{~g}-\mathrm{i}\right)$. Consistent with findings of Blackmon et al. (1979),
SAT and SLP tend to be negatively correlated in the vicinity of the reference grid points in these regions. The patterns also indicate that SAT tends to be higher when the juxtaposition of SLP and SAT is indicative of anomalous warm advection and vice versa. The regression patterns shown in Fig. 2 are analogous to the independent variable loading patterns $(\mathbf{P})$, which are not identical to the cross-correlation maps that are used as weighting functions (W) to develop the PLS predictor time series. 
TABLE 1. Fraction of monthly Northern Hemisphere $\left(20^{\circ}-90^{\circ} \mathrm{N}\right)$ cold season SAT variance explained (\%) by the indicated methodologies: (from left to right) pointwise PLS regression based on $n$ SLP-based predictors formed from $120^{\circ}$ predictor fields, PC-wise PLS regression based on 40 SAT PCs and $n$ SLP-based predictors formed from hemispheric predictor fields, fieldwise PLS regression based on $n$ SLP-based predictors formed from hemispheric predictor fields, and principal component regression, a multiple linear regression using the leading $n$ PCs of NH cold season SLP. SAT data from the land-only GHCN dataset; SLP data from the 20CR product.

\begin{tabular}{ccccc}
\hline \hline$n$ passes & Pointwise PLSR & PC-wise PLSR & Fieldwise PLSR & Pointwise PCR \\
\hline 1 & 36 & 34 & 9 & 9 \\
2 & 44 & 43 & 16 & 15 \\
3 & 47 & 47 & 23 & 22 \\
4 & 50 & 50 & 30 & 24 \\
5 & 51 & 51 & 32 & 30 \\
6 & 53 & 53 & 35 & 32 \\
7 & 54 & 54 & 37 & 34 \\
8 & 55 & 55 & 39 & 37 \\
9 & 56 & 55 & 40 & 39 \\
\hline
\end{tabular}

The amplitude of the patterns in Fig. 2 represents the regression coefficient of local SLP upon the standardized PLS predictors and is thus proportional to the fraction of the local SLP variance that they explain, not to the fraction of predictand SAT variance that they explain.

\section{b. Fieldwise dynamical adjustment}

In section $3 \mathrm{a}$ we considered the dynamical adjustment of individual SAT time series. Here we consider the dynamical adjustment of the entire SAT field. The adjustment can be performed one grid point at a time, which we will continue to refer to as the pointwise approach. A computationally efficient way of performing the adjustment is to decompose the predictand (SAT) field into EOFs and to perform PLS regression upon a truncated set of the corresponding PCs. The dynamically adjusted SAT field is formed by summing the outer products of the truncated set of SAT PCs with their respective EOFs, appropriately scaled by the square root of the corresponding eigenvalues. This methodology, which we will refer to as PC-wise dynamical adjustment, is applied to the GHCN NH SAT field in Smoliak (2013). A more direct way of dynamically adjusting the entire NH SAT field is to use the multivariate predictand approach described earlier in this section. We will refer to this as the fieldwise dynamical adjustment.

The first three columns in Table 1 compare the fraction of cold season NH SAT variance explained by applying pointwise, $\mathrm{PC}$-wise, and fieldwise PLS regression dynamical adjustment. It is evident that using the pointwise and PC-wise approaches it is possible to explain nearly half the variance of the SAT field using only three SLP predictors. They are more parsimonious than the fieldwise approach, which requires more predictors to explain a comparable fraction of the variance. The fourth column of Table 1 shows the fraction explained by the leading PCs of the cold season NH SLP field, one of the approaches used previously by Thompson et al. (2009). It is clear that the pointwise and PC-wise PLS regression approaches are also more parsimonious than $\mathrm{PC}$ regression.

The pattern of NH cold season SAT variance (Fig. 3a) is characterized by a meridional gradient with the largest values over the high-latitude NH continents. Figures $3 b, d, f, h$ compare the fraction of variance explained by pointwise PLS regression with 3 PLS predictors, PCwise PLS regression retaining the 40 leading SAT PCs with 3 PLS predictors, fieldwise PLS regression with 10 PLS predictors, and PC regression using the leading 10 PCs of NH cold season SLP. The largest local values of the fraction of variance explained are seen over the continental interiors, particularly just to the north of regions of high mountain ranges, suggestive of the influence of orography on airmass formation and movement, as well as the orientation of the climatological mean isotherms. The patterns are all qualitatively similar, but all three variants of PLS regression explain slightly larger fractions of variance than $\mathrm{PC}$ regression. Residual variance maps shown in Figs. 3c,e,g,i exhibit the highest variance along the Arctic coastline. This variance may be attributable to dynamical patterns left unexplained, or to thermodynamical processes. For example, model simulations of global warming in the twenty-first century by Deser et al. (2010) and Alexander et al. (2010) illustrate how sea ice concentration and snow extent changes influence SAT, and imply that year-to-year changes in sea ice concentration or snow extent could have contributed to SAT variability over the twentieth century.

While PCs have been demonstrated to be an effective means of adjusting hemispheric and global-mean SAT time series (Thompson et al. 2009), it is clear that PLS regression is superior at the gridpoint scale (Fig. 3) as 

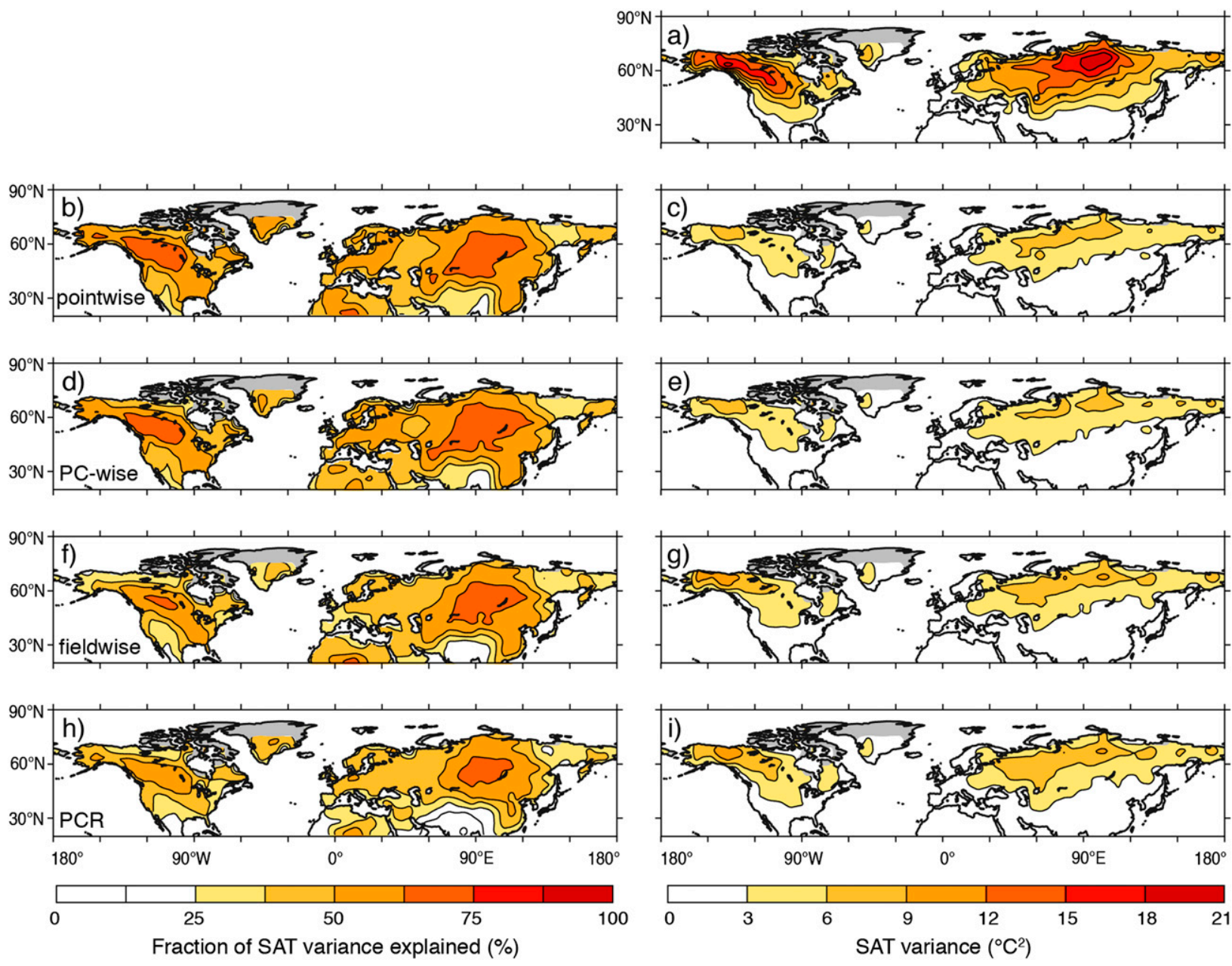

FIG. 3. Comparison of (a) Northern Hemisphere $\left(20^{\circ}-90^{\circ} \mathrm{N}\right)$ cold season SAT variance with (left) the fraction of variance explained $(\%)$ and (right) residual NH cold season SAT variance resulting from dynamical adjustments using (b), (c) pointwise PLS regression based on 3 SLP-based predictors formed from $120^{\circ}$ predictor fields; (d),(e) PC-wise PLS regression based on 40 SAT PCs and 3 SLP-based predictors formed from hemispheric predictor fields; (f),(g) fieldwise PLS regression based on 10 SLP-based predictors formed from hemispheric predictor fields; and (h),(i) principal component regression based on the leading 10 PCs of NH cold season SLP.

well as in an area-average sense (Table 1). The next section describes how PLS regression may be used to estimate the dynamical contribution to SAT trends.

\section{Application to the attribution of surface air temperature trends}

To illustrate how dynamical adjustment can be used in the attribution of trends in the NH SAT field, we apply the four previously described approaches to the boreal cold season trends during two historical reference periods: $1965-2000$, an interval marked by rapid warming over the Northern Hemisphere continents poleward of $40^{\circ} \mathrm{N}$, and $1920-2011$, a longer period spanning the entire data record used in this article. Following Wallace et al. (2012), we will focus on land areas poleward of $40^{\circ} \mathrm{N}$ (N SAT) when quantifying the dynamical contributions to SAT trends. The dynamical contribution to the SAT trend is defined as the difference between SAT trends calculated from unadjusted and adjusted data. Our so-called "residual" SAT trends are based on the dynamically adjusted data.

The SLP tendency during the interval 1965-2000 based on ordinary least squares best fit is shown in Fig. 4a. In agreement with prior studies (Hurrell 1996; Thompson et al. 2000; Quadrelli and Wallace 2004) it is characterized by a strong trend toward the high index polarity of the NAM and the PNA pattern, the latter occurring in association with a regime shift in the $\mathrm{Pa}$ cific decadal oscillation (PDO) toward its positive polarity (Quinn and Neal 1984; Nitta and Yamada 1989; Zhang et al. 1997; Mantua et al. 1997). The spatial 
1965-2000
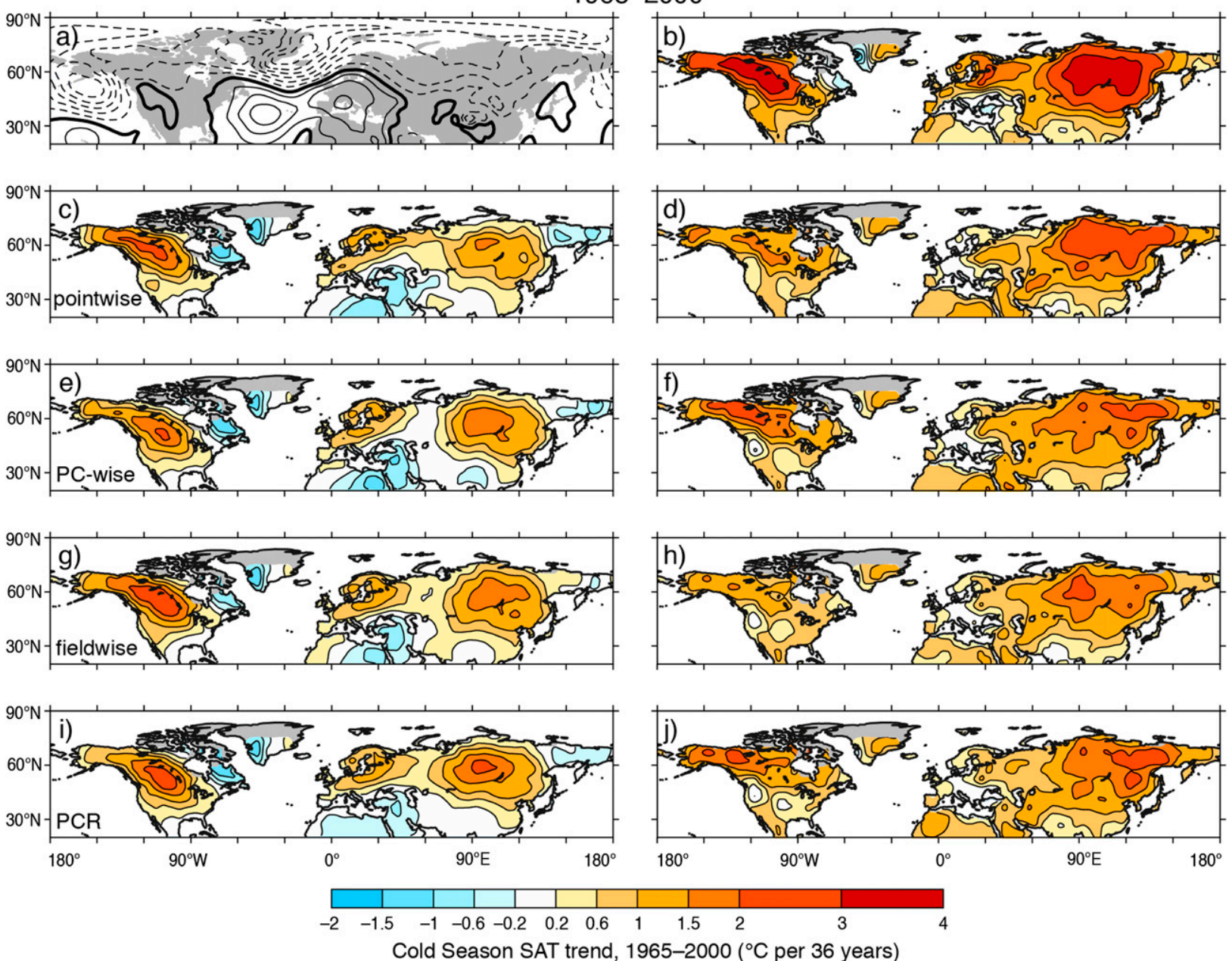

FIG. 4. (a) Northern Hemisphere $\left(20^{\circ}-90^{\circ} \mathrm{N}\right)$ cold season SLP trends $1965-2000$, based on ordinary least squares best fit, contour interval $1 \mathrm{hPa}(36 \mathrm{yr})^{-1}$, negative contours dashed, zero contour thickened. (b) SAT trends 1965-2000, based on ordinary least squares best fit $\left[{ }^{\circ} \mathrm{C}(36 \mathrm{yr})^{-1}\right]$. (c)-(j) (left) Dynamical contribution to SAT trends and (right) residual SAT trends derived from the four dynamical adjustment schemes described in Fig. 3.

pattern of the SAT linear trend during this interval, shown in Fig. 4b, exhibits a strong enhancement of the warming over the continental interiors at latitudes poleward of $40^{\circ} \mathrm{N}$.

The four dynamical adjustment schemes considered in this section yield similar dynamical contributions to the SAT trends poleward of $40^{\circ} \mathrm{N}$ during $1965-2000$, both in terms of the spatial pattern (Figs. 4c,e,g,i) and magnitude (Table 2). The spatial pattern of the dynamical adjustment is characterized by a tendency to warm the continental interiors and to cool portions of the Canadian shield, Greenland, and the Sahara. The dynamical contribution to the boreal cold season SAT trend averaged over the land area poleward of $40^{\circ} \mathrm{N}$ during 1965 2000 is approximately $0.7^{\circ} \mathrm{C}$, about $40 \%$ of the raw boreal cold season trend $\left(1.72^{\circ} \mathrm{C}\right)$. None of the approaches is very sensitive to the number of predictors included (Table 2), or to the areal extent of the predictor field (Smoliak 2013). That the dynamical contribution to the SAT trend poleward of $40^{\circ} \mathrm{N}$ levels off after three PCs are included in the PC regression scheme suggests that a large fraction of the dynamical contribution comes from the three leading EOFs of $\mathrm{NH}$ cold season SLP, which encompass the major Northern Hemisphere wintertime teleconnection patterns (Thompson et al. 2000; Quadrelli and Wallace 2004; Smoliak 2009).

The patterns of residual SAT trends shown in Figs. 4d,f,h,j share much in common. Whereas the warming in the pattern of unadjusted $\mathrm{NH}$ cold season SAT trends is characterized by maxima in the continental interiors (Fig. 4b), the maxima in the patterns of residual SAT trends are centered farther north, along the Arctic 
TABLE 2. Comparison of the dynamical contribution $\left[{ }^{\circ} \mathrm{C}(36 \mathrm{yr})^{-1}\right]$ to the rise in boreal cold season N SAT, 1965-2000, an average over land areas poleward of $40^{\circ} \mathrm{N}$, as estimated from the dynamically adjusted NH SAT field $\left(20^{\circ}-90^{\circ} \mathrm{N}\right)$ using (from left to right) pointwise PLS regression based on $n$ SLP-based predictors formed from $120^{\circ}$ predictor fields, PC-wise PLS regression based on 40 SAT PCs and $n$ SLPbased predictors formed from hemispheric predictor fields, fieldwise PLS regression based on $n$ SLP-based predictors formed from hemispheric predictor fields, and principal component regression, a multiple linear regression using the leading $n$ PCs of NH cold season SLP. Data sources as in Table 1.

\begin{tabular}{ccccc}
\hline \hline$n$ passes & Pointwise PLSR & PC-wise PLSR & Fieldwise PLSR & Pointwise PCR \\
\hline 1 & 0.75 & 0.77 & 0.53 & 0.52 \\
2 & 0.70 & 0.68 & 0.58 & 0.83 \\
3 & 0.70 & 0.71 & 0.61 & 0.76 \\
4 & 0.72 & 0.69 & 0.75 & 0.74 \\
5 & 0.72 & 0.67 & 0.73 & 0.78 \\
6 & 0.72 & 0.65 & 0.77 & 0.77 \\
7 & 0.73 & 0.68 & 0.73 & 0.77 \\
8 & 0.76 & 0.76 & 0.77 & 0.77 \\
9 & 0.76 & 0.73 & 0.76 & 0.78 \\
10 & 0.76 & 0.74 & & 0.78 \\
\hline
\end{tabular}

coast. The pattern of residual SAT trends associated with the PC regression adjustment is somewhat different than the other three, with slightly less residual warming over central Siberia. Otherwise it bears a strong resemblance to the estimates from the three variants of PLS regression.

The NH cold season SLP trends over the period 19202011 (Fig. 5a) are not as large as over the reference interval 1965-2000, and the spatial structures are different. Whereas the 1965-2000 period is characterized by a strengthening of SLP centers of action over the NH oceans (the Icelandic low, the Aleutian low, and the Azores high), the 1920-2011 period is characterized by pressure changes over the interior of the high-latitude continents. The largest NH cold season SAT rises over the extended interval (Fig. 5b) are roughly comparable to those for the 1965-2000 reference period, but they are spread over a time interval more than twice as long, so the trends in degrees Celsius per decade are much smaller. The spatial pattern of the trends is similarly characterized by a warming over the interior of the continents, although the warming maximum over Eurasia is shifted slightly to the south of the corresponding maximum for the interval 1965-2000. The patterns of dynamical contributions to the warming yielded by the four approaches shown in Figs. 5c,e,g,i are similar, although fieldwise PLS regression accounts for a much larger portion of the high-latitude warming. The fact that fieldwise PLS regression explains a large fraction of the trend over the same period that the dynamical adjustment is calculated raises the concern that it may be more susceptible to fitting trends in the two fields than the other approaches and is therefore not as reliable as the other methods. We tested high-pass filtering the SAT field prior to calculating successive fieldwise PLS regression predictors. This additional step reduced its tendency to fit trends in the input fields over the extended period, but also reduced the fraction of NH SAT variance explained (not shown), making it less attractive than the pointwise and PC-wise variants of PLS regression. With the exception of the fieldwise PLS regression results, the patterns of residual SAT trends are similar (Figs. 5d,f,j). The dynamical contribution to the boreal cold season SAT trend averaged over the land area poleward of $40^{\circ} \mathrm{N}$ during $1920-2011$ is approximately $0.7^{\circ} \mathrm{C}$, about $40 \%$ of the raw boreal cold season trend $\left(1.73^{\circ} \mathrm{C}\right)$.

Because the dynamical contribution to NH SAT trends is appreciable and tends to be concentrated in the boreal cold season, the hemispheric-mean SAT time series for the cold and warm seasons do not track on another very closely, as shown in the upper portion of Fig. 6a. Applying a dynamical adjustment to the NH cold season SAT field before computing the hemispheric-mean SAT smooths the cold season time series and brings it into much closer alignment with the warm season time series, as shown in the lower portion of Fig. 6a. The dynamical adjustment has an even stronger influence on regional time series, as illustrated in Fig. 6b (continental United States) and Fig. 6c (Irkutsk Oblast, Russia). For all three domains shown in Fig. 6, the dynamical contribution to the boreal cold season warming trend over the 92-yr record 1920-2011 is quite substantial.

\section{Discussion and summary}

The dynamical adjustment protocols described in this article incorporate and extend the set of schemes that have been proposed over the past 20 years. We have shown that dynamical adjustment of the NH SAT field based on SLP can be used for climate diagnostic studies of both short-term climate fluctuations and long-term 

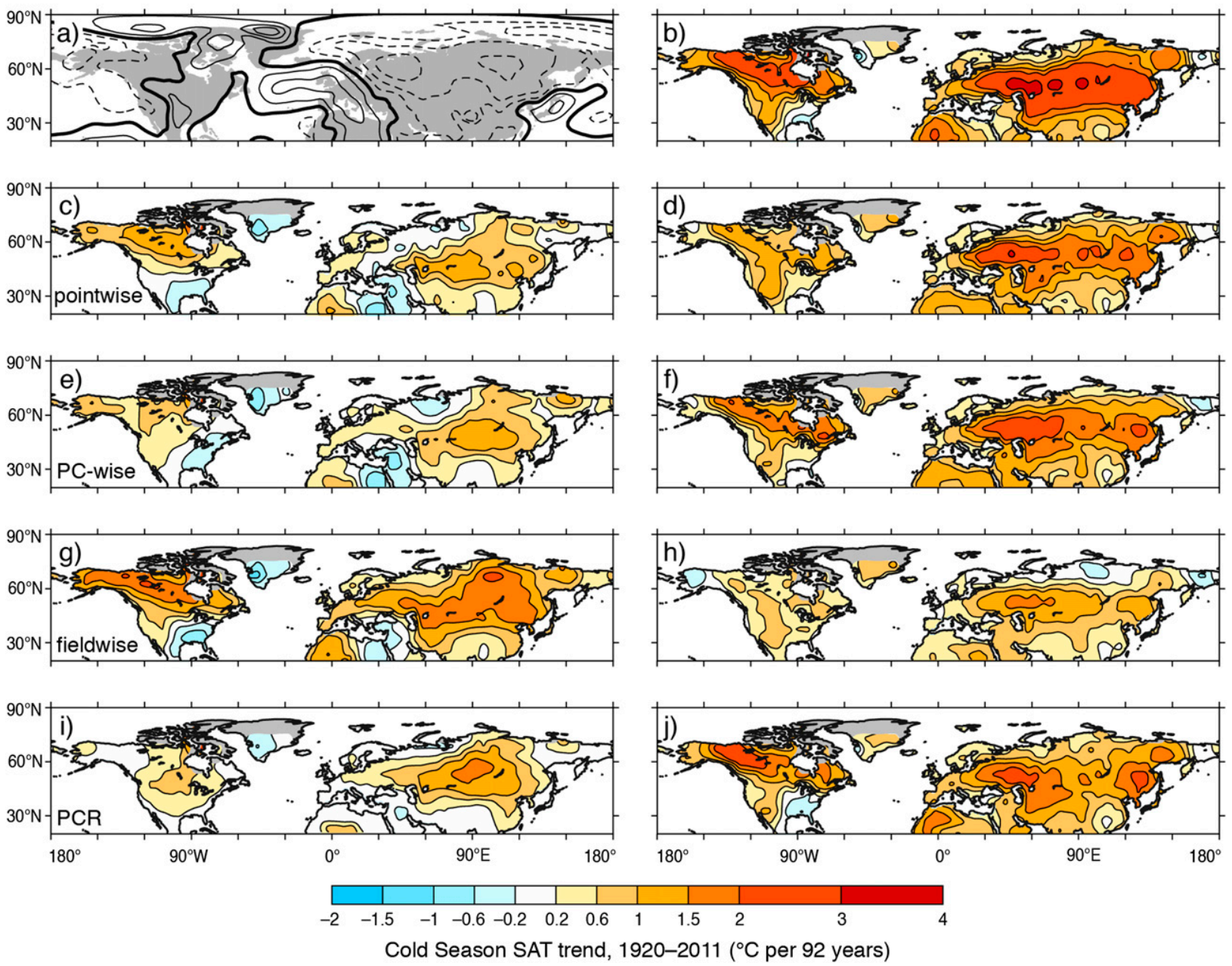

FIG. 5. As in Fig. 4, but for trends of (a) SLP [hPa $(92 \mathrm{yr})^{-1}$ ] and (b)-(j) SAT [ ${ }^{\circ} \mathrm{C}(92 \mathrm{yr})^{-1}$ ] for $1920-2011$.

trends. The adjustment removes most of the sampling variability introduced by internally generated variations in the atmospheric circulation such as the annular modes. Here we have considered only the SAT variations induced by NH SLP variability during the boreal cold season. It is conceivable that some additional sampling variability could be removed by taking into account the weaker dynamical influence of variations in the boreal warm season $\mathrm{NH}$ circulation, as well as the influence of the Southern Hemisphere (SH) circulation on SH SAT. Further refinement of the climate change signal in the global SAT record could be achieved by incorporating an additional dynamical adjustment designed to remove the year-to-year variability associated with ENSO (Santer et al. 2001; Thompson et al. 2009; Foster and Rahmstorf 2011).

We have shown that the pointwise and PC-wise approaches to PLS regression are able to explain a larger fraction of NH SAT variance with fewer predictors and are thus preferable to fieldwise PLS regression and PC regression for the applications considered in this study. To further illustrate the superiority of the PLS-based approach, we repeated the pointwise PLS regression-based dynamical adjustment using a set of "reduced" SLP fields, the first based on its leading EOF, the second based on its leading two EOFs, etc. the results are summarized in Table 3. For a reduced SLP field of any specified truncation, the $\mathrm{PC}$ regression approach to dynamical adjustment should account for the maximum possible fraction of the variance. It is evident that the dynamical adjustments based on PLS regression are almost as large as those based on PC regression. It follows that the superior performance of pointwise and PC-wise PLS regression is mainly due to the fact that they are able to exploit the information inherent in all EOFs without being susceptible to overfitting. 


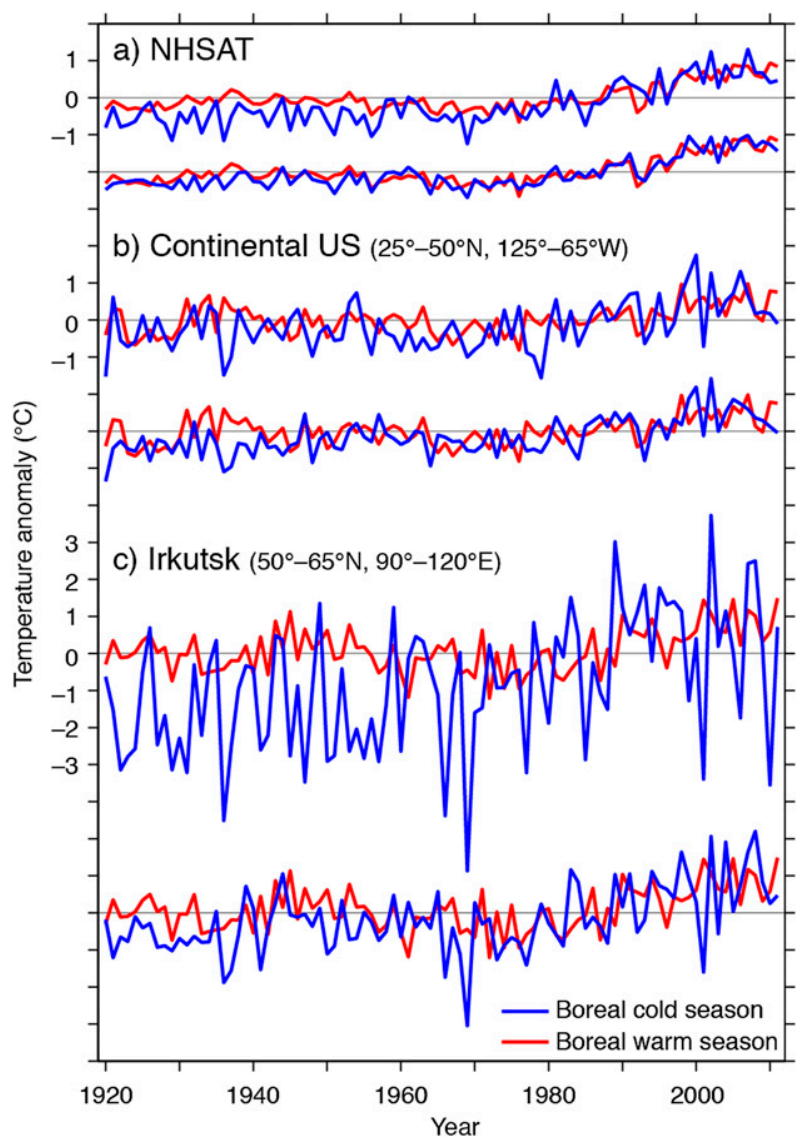

FIG. 6. (a) Seasonal-mean, NH-mean SAT over land areas poleward of $20^{\circ} \mathrm{N}$ for the boreal cold season (blue, NovemberApril) and the boreal warm season (red, May-October), 19202011, based on the land-only GHCN dataset, (top) before and (bottom) after dynamically adjusting the cold season SAT field using PLS regression in a pointwise manner as described in the text. (b) As in (a), but for an area average over land areas spanning the continental United States $\left(25^{\circ}-50^{\circ} \mathrm{N}, 125^{\circ}-65^{\circ} \mathrm{W}\right)$. (c) As in (a), but for an area average over land areas in central Eurasia, around the Russian Federation of Irkutsk Oblast $\left(50^{\circ}-65^{\circ} \mathrm{N}, 90^{\circ}-120^{\circ} \mathrm{E}\right)$. Each tick mark on the abscissa indicates $1^{\circ} \mathrm{C}$. Anomalies are displayed with respect to the base period 1971-2000.

We tested the sensitivity of the fraction of SAT variance explained by PC regression to the choice of whether or not to standardize the SLP field. The explained variance based on the leading 10 PCs of the standardized SLP field is generally smaller than the values based on EOFs of the raw SLP field. This result seems plausible, given that the 10 leading PCs of the raw SLP field explain a substantially larger fraction of its variance than the leading 10 PCs of the standardized SLP field. When the leading 20 PCs are retained in the PC regression scheme, the variances explained by the EOFs of the raw and standardized SLP field are almost identical.
The PLS regression scheme used in this study is suboptimal in the sense that we retain only three PLS predictors for each grid point or SAT PC, whereas the number of PLS predictors retained for each grid point or SAT PC may be greater. Our choice of three PLS predictors is conservative, but to justify the use of additional passes would require extensive cross validation (see the appendix for further discussion). Hence, there is scope for improving the performance of PLS regression in explaining the variance of the SAT field, but to do so would require steps to ensure the robustness of the results.

In deriving a dynamical adjustment using any technique involving linear regression, it is possible that SLP patterns could be unduly influenced by trends and multidecadal variability that are more appropriately viewed as an integral part of the global warming "signal" than as part of the dynamically or thermodynamically induced "noise." To ensure that the SLP-based PLS predictors of SAT are not susceptible to fitting trends, we high-pass filtered the target SAT time series prior to calculating cross-correlation patterns. This assures that the SLP patterns used to form PLS predictors are based on high-frequency relationships between SLP and SAT. Trends and multidecadal variability are thus reflected in the SLP-based PLS predictors of SAT only to the extent that the cross-correlation patterns project onto low-frequency SLP patterns. We explored various high-pass filters and found that the results were not sensitive to the choice of method (see the appendix for additional details).

This study presented dynamical adjustment with PLS regression as a development in the application of regressionbased methods in climate diagnostics; non-regression-based linear diagnostic methods have also been advanced in recent years (e.g., with respect to the problem of identifying and removing ENSO-related variability from the sea surface temperature field) (Penland and Matrosava 2006; Compo and Sardeshmukh 2010). Extending dynamical adjustment applications to non-regression-based methods is beyond the scope of this study.

We have presented evidence that dynamical adjustment is capable of separating dynamically induced SAT trends from thermodynamically or radiatively induced SAT trends. However, the methodology we described makes no distinction between externally forced and internally generated contributions to SAT or SLP variability and trends. Separating signal and noise using observations alone is at best extremely difficult, requiring a variety of assumptions about the climate system response to external forcing and its internal variability (Barnett et al. 1999; Hegerl and Zwiers 2011). To address these issues, general circulation models (GCMs) have 
TABLE 3. Comparison of statistics associated with dynamical adjustment using pointwise PLS regression with three passes and principal component regression with the leading $n$ PCs of NH cold season SLP, based on a reduced space predictor dataset defined by the NH SLP field poleward of $20^{\circ} \mathrm{N}$ truncated at the EOF indicated in the far left column. NH SAT variance explained is based upon an average over land areas poleward of $20^{\circ} \mathrm{N}$. The dynamical contribution is specific to N SAT, an average over land areas poleward of $40^{\circ} \mathrm{N}$. Data sources as in Table 1.

\begin{tabular}{|c|c|c|c|c|}
\hline \multirow[b]{2}{*}{ EOF truncation } & \multicolumn{2}{|c|}{ NH SAT variance explained (\%) } & \multicolumn{2}{|c|}{ Dynamical contribution $\left[{ }^{\circ} \mathrm{C}(36 \mathrm{yr})^{-1}\right]$} \\
\hline & Pointwise PLSR & Pointwise PCR & Pointwise PLSR & Pointwise PCR \\
\hline 1 & 9 & 9 & 0.52 & 0.52 \\
\hline 2 & 15 & 15 & 0.83 & 0.83 \\
\hline 3 & 22 & 22 & 0.76 & 0.76 \\
\hline 4 & 24 & 24 & 0.74 & 0.74 \\
\hline 5 & 26 & 26 & 0.79 & 0.78 \\
\hline 6 & 29 & 30 & 0.76 & 0.77 \\
\hline 7 & 31 & 32 & 0.76 & 0.77 \\
\hline 8 & 33 & 34 & 0.74 & 0.77 \\
\hline 9 & 35 & 37 & 0.75 & 0.78 \\
\hline 10 & 37 & 39 & 0.74 & 0.78 \\
\hline No truncation & 47 & - & 0.70 & - \\
\hline
\end{tabular}

been used to estimate the climate system response to external forcings and the magnitude of the internal variability (Santer et al. 1995; Gillett et al. 2002; Meehl et al. 2007a; Hegerl et al. 2007), as well as the role of internal variability in shaping regional trends over time (Deser et al. 2012a,b). For instance, single-model large ensemble integrations have been used to separate simulated SAT and precipitation trends into externally forced and internally generated components (Deser et al. 2014). Dynamical adjustment could be applied to single-model large ensembles to further decompose the externally forced and internally generated components of the simulated trends into dynamically induced and thermodynamically induced components. This additional level of attribution will facilitate physical insights on the nature of regional climate change.

Acknowledgments. We thank Clara Deser, David W. J. Thompson, and Mark T. Stoelinga for their methodological insights and constructive feedback at various stages of this research. We would also like to acknowledge the helpful comments and suggestions of two anonymous reviewers. BVS and JMW were supported by the Climate Dynamics Program Office of the National Science Foundation under Grant 0812802. QF was supported by the NASA Grant NNX13AN49G.

\section{APPENDIX}

\section{Methodological Considerations for PLS Regression}

In this appendix we address a series of methodological considerations concerning the implementation of PLS regression. They include (i) the choice of the number of predictors to be retained in the dynamical adjustment scheme, (ii) whether it is better to apply the procedure to the SAT time series for every grid point or to a designated area-average SAT time series, and (iii) the choice of the areal extent of the predictor field.

\section{a. Are the results sensitive to the choice of the number of PLS predictors retained in the adjustment scheme?}

In principle, PLS regression can be repeated until the PLS predictors cease to explain appreciable fractions of the variance. However, in practice, as a result of the finite sample size, the fraction of variance explained by successive PLS predictors never quite levels off. For example, in the first column of Table A1 the first few predictors account for most of the explained variance but the continuing rise in the fraction of explained variance out to 10 predictors raises concerns about overfitting (Davis 1976). Hence, the decision of how many cross-correlation patterns to use as predictors of the reference time series should be determined by cross validation.

Here we perform cross validation by deriving dynamical adjustments for one cold season at a time, using cross-correlation patterns derived from the remaining years. Following this approach, often referred to as the "leave $p$ out" method, we can construct a dynamical adjustment time series for each cold season based on independent SLP data, and combine the adjustments for the entire period of record to obtain a cross-validated residual. Employing cross validation reduces the tendency of the fraction of variance to rise, as shown in the second column of Table A1, but does not eliminate it completely. The resilience of the upward creep in 
TABLE A1. Fraction of monthly-mean cold season NH-mean $\left(20^{\circ}-90^{\circ} \mathrm{N}\right.$, NHSAT) SAT variance explained (\%) by $n$ SLP-based PLS predictors based on unfiltered and high-pass (HP)-filtered input SAT data, with and without leave- $p$-out cross validation (CV). See the appendix text for details. SAT data from the land-only GHCN dataset; SLP data from the 20CR product.

\begin{tabular}{ccccc}
\hline \hline$n$ passes & Unfiltered, no CV & Unfiltered, CV & HP filtered, no CV & HP filtered, CV \\
\hline 1 & 41 & 39 & 38 & 38 \\
2 & 51 & 48 & 44 & 44 \\
3 & 59 & 54 & 51 & 47 \\
4 & 63 & 57 & 52 & 49 \\
5 & 66 & 59 & 54 & 50 \\
6 & 68 & 60 & 54 & 50 \\
7 & 70 & 61 & 55 & 50 \\
8 & 71 & 61 & 55 & 51 \\
9 & 72 & 62 & 55 & 51 \\
10 & 74 & 62 & & \\
\hline
\end{tabular}

explained variance is likely due to the existence of yearto-year autocorrelation in the SLP and SAT time series. We tested leaving out multiple years of data to further reduce the autocorrelation and found similar results, even when leaving out periods of a decade or more (Smoliak 2013). To test whether or not our dynamical adjustment scheme is unduly influenced by multidecadal trends in the data, we applied our dynamical adjustment procedure using high-pass-filtered SAT data to generate the cross-correlation patterns in the SLP field. The data were filtered by removing the cold season mean from the mean for each month within a given cold season, thereby retaining only the intraseasonal month-to-month variability. We also experimented with various high-pass Lanczos filters with low-frequency cutoffs at periods of $20,10,5,3$, and $1 \mathrm{yr}$. We found the results to be insensitive to the specific method of high-pass filtering employed. Filtering the data in this manner further reduces the tendency for the fraction of variance explained to creep upward as more and more predictors are added, as shown in the third column of Table A1, as does performing cross validation on dynamical adjustment time series generated from cross-correlation functions based on high-passfiltered data (Table A1, fourth column). See Smoliak (2013) for additional analysis.

\section{b. Is the area average of the dynamical adjustment the same as the dynamical adjustment of the area average?}

The answer, at least in principle, is yes, by virtue of the fact that the spatial averaging operator and the matrix operations to compute the dynamical contribution are linear and thus commutative, that is,

$$
\left\langle\mathbf{P}\left\langle\mathbf{P}^{\mathrm{T}} \mathbf{T}\right\rangle\right\rangle=\left\langle\left\langle\mathbf{T}^{\mathrm{T}} \mathbf{P}\right\rangle \mathbf{P}^{\mathrm{T}}\right\rangle,
$$

where $\mathbf{P}$ and $\mathbf{T}$ are standardized SLP and SAT fields, respectively, and angle brackets represent the spatial average operator. The left-hand side of (A1) represents the dynamical contribution to the area average of the $\mathbf{T}$ field, and the right-hand side represents the area average of the dynamical contribution to the $\mathbf{T}$ field. If the data matrix is incomplete, the identity in (A1) may not hold exactly and the dynamical adjustment may exhibit some

TABLE A2. Fraction of Northern Hemisphere $\left(20^{\circ}-90^{\circ} \mathrm{N}\right)$ cold season SAT variance explained (\%) by pointwise PLS regression of the NH SAT monthly anomaly field with $n$ SLP-based predictors (rows) using SLP predictor fields of various areal extents (columns, latitude and longitude indicated in header). Data sources as in Table A1.

\begin{tabular}{cccccccccc}
\hline \hline & \multicolumn{7}{c}{ Extent (lat $\times$ lon) } \\
\cline { 2 - 9 }$n$ passes & $5^{\circ} \times 5^{\circ}$ & $10^{\circ} \times 10^{\circ}$ & $30^{\circ} \times 30^{\circ}$ & $60^{\circ} \times 60^{\circ}$ & $70^{\circ} \times 90^{\circ}$ & $70^{\circ} \times 120^{\circ}$ & $70^{\circ} \times 150^{\circ}$ & $70^{\circ} \times 180^{\circ}$ & $70^{\circ} \times 360^{\circ}$ \\
\hline 1 & 17 & 19 & 29 & 35 & 36 & 36 & 36 & 36 \\
2 & 35 & 34 & 39 & 43 & 44 & 44 & 44 & 44 & 44 \\
3 & 40 & 39 & 44 & 46 & 47 & 47 & 48 & 48 & 48 \\
4 & 43 & 42 & 47 & 49 & 49 & 50 & 50 & 50 & 50 \\
5 & 45 & 44 & 49 & 51 & 51 & 51 & 52 & 52 & 53 \\
6 & 47 & 45 & 51 & 53 & 53 & 53 & 53 & 55 \\
7 & 50 & 47 & 53 & 54 & 54 & 54 & 55 & 55 \\
8 & 54 & 48 & 54 & 55 & 55 & 55 & 56 & 56 \\
9 & 60 & 49 & 55 & 56 & 56 & 56 & 57 & 57 & 56 \\
10 & 68 & 50 & 56 & 57 & 57 & 57 & 58 & 58 & 58 \\
\hline
\end{tabular}


TABLE A3. Fraction of monthly cold season SAT variance explained (\%) by three SLP-based predictors from PLS regression at two sample grid points, distinguishing the contributions from each predictor $\left(Z_{1}, Z_{2}\right.$, and $\left.Z_{3}\right)$, varying the NH SLP predictor field size as indicated (degrees latitude by degrees longitude, centered longitudinally over the reference grid point, within a latitudinal range of $20^{\circ}-$ $90^{\circ} \mathrm{N}$ ). Results correspond to the SLP patterns shown in Figs. S1 and S2 of the supplementary material. Data sources as in Table A1.

\begin{tabular}{rcrrrrrrrrr}
\hline & Extent (lat $\times$ lon) & $5^{\circ} \times 5^{\circ}$ & $10^{\circ} \times 10^{\circ}$ & $30^{\circ} \times 30^{\circ}$ & $60^{\circ} \times 60^{\circ}$ & $70^{\circ} \times 90^{\circ}$ & $70^{\circ} \times 120^{\circ}$ & $70^{\circ} \times 150^{\circ}$ & $70^{\circ} \times 180^{\circ}$ & $70^{\circ} \times 360^{\circ}$ \\
\hline $60^{\circ} \mathrm{N}, 100^{\circ} \mathrm{E}$ & $Z_{1}$ & 39 & 41 & 57 & 62 & 63 & 63 & 62 & 61 \\
& $Z_{2}$ & 17 & 18 & 6 & 2 & 3 & 3 & 5 & 7 \\
& $Z_{3}$ & 1 & 3 & 3 & 2 & 2 & 3 & 3 & 2 \\
& Total & 57 & 62 & 66 & 66 & 68 & 69 & 70 & 70 & 70 \\
$60^{\circ} \mathrm{N}, 115^{\circ} \mathrm{W}$ & & & & & & & & & & \\
& $Z_{1}$ & 39 & 38 & 38 & 43 & 51 & 54 & 53 & 52 & 49 \\
& $Z_{2}$ & 15 & 15 & 22 & 21 & 15 & 11 & 12 & 12 & 9 \\
& $Z_{3}$ & 4 & 7 & 4 & 5 & 3 & 3 & 3 & 3 \\
& Total & 58 & 60 & 64 & 69 & 69 & 68 & 68 & 67 & 66 \\
\hline
\end{tabular}

dependence on whether the spatial averaging is applied before or after the calculation of the dynamical contribution. Notwithstanding this caveat, the identity in (A1) implies that if the goal of dynamical adjustment is to remove dynamical contributions to the variability of an area-average time series, there is no benefit to performing a pointwise dynamical adjustment. The pointwise approach is useful in its own right, however, because it yields a dynamically adjusted field as a by-product. Furthermore, in the next subsection we will show that in some cases customized regional-scale predictor fields are more effective at explaining local SAT variance than hemispheric predictor fields.

\section{c. Are the results sensitive to the choice of the areal extent of the predictor field?}

When adjusting the NHSAT time series, the entire NH SLP field poleward of $20^{\circ} \mathrm{N}$ is used to capture SLP patterns that influence SAT variability around the whole hemisphere. When adjusting individual gridpoint time series, however, it is not as clear how large a predictor field to use. To test the sensitivity of the pointwise PLS regression results to the choice of the areal extent of the predictor field, we repeated the pointwise PLS regression procedure for the NH SAT field using progressively smaller predictor fields centered over the reference grid point, from the entire $\mathrm{NH}$ poleward of $20^{\circ} \mathrm{N}$ down to $5^{\circ}$ latitude by $5^{\circ}$ longitude areas.

We found that the fraction of NH SAT variance explained by the first PLS predictor increases as the areal extent of the predictor field increases, up to a longitudinal width of $90^{\circ}$, after which it remains nearly flat, as shown in the top row of Table A2. This result also holds when additional PLS predictors are added to the dynamical adjustment scheme, as shown in subsequent rows. Table A3 shows the fraction of local SAT variance explained by dynamical adjustment based on three PLS predictors for the two sample grid points shown in Fig. 2. The results presented in Table A3 indicate that although the fraction of variance explained by individual patterns fluctuates somewhat with domain size, the total amount of variance explained by the leading three patterns does not. This result suggests that the dynamical adjustment exhibits very little sensitivity to the areal extent of the predictor domain, implying that most of the important information related to SAT variability at most reference grid points is contained in the SLP field within a $90^{\circ}$ longitudinal sector.

The SLP regression patterns are relatively insensitive to the areal extent of the predictor field, especially for sizes in the range of $30^{\circ}-180^{\circ}$ of longitude (see Figs. S1 and $\mathrm{S} 2$ in the supplemental material). Domains smaller than $30^{\circ}$ of longitude do not fully capture the far-field features, which evidently contribute to the predictive skill. Domain sizes larger than $180^{\circ}$ of longitude in extent are problematical because in the absence of constraints the patterns tend to spread over the entire hemisphere and become more difficult to interpret.

\section{REFERENCES}

Abdi, H., 2010: Partial least squares regression and projection on latent structure regression (PLS regression). WIREs Comput. Stat., 2, 97-106, doi:10.1002/wics.51.

Alexander, M. A., R. Tomas, C. Deser, and D. M. Lawrence, 2010: The atmospheric response to projected terrestrial snow changes in the late twenty-first century. J. Climate, 23, 64306437, doi:10.1175/2010JCLI3899.1.

Barnett, T. P., and R. Preisendorfer, 1987: Origins and levels of monthly and seasonal forecast skill for United States surface air temperatures determined by canonical correlation analysis. Mon. Wea. Rev., 115, 1825-1850, doi:10.1175/ 1520-0493(1987)115<1825:OALOMA $>2.0$. CO;2.

— , and Coauthors, 1999: Detection and attribution of recent climate change: A status report. Bull. Amer. Meteor. Soc., 80, 2631-2660, doi:10.1175/1520-0477(1999)080<2631: DAAORC $>2.0 . \mathrm{CO} ; 2$.

Blackmon, M. L., R. A. Madden, J. M. Wallace, and D. S. Gutzler, 1979: Geographical variations in the vertical structure of 
geopotential height fluctuations. J. Atmos. Sci., 36, 2450-2466, doi:10.1175/1520-0469(1979)036<2450:GVITVS>2.0.CO;2.

Bradley, R. S., H. F. Diaz, P. D. Jones, and P. M. Kelly, 1987: Secular fluctuations in temperature over the Northern Hemisphere land areas and mainland China since the mid-19th century. The Climate of China and Global Climate, D. Yeh et al., Eds., China Press and Springer Verlag, 77-87.

Braganza, K., D. J. Karoly, A. C. Hirst, M. E. Mann, P. A. Stott, R. J. Stouffer, and S. F. B. Tett, 2003: Simple indices of global climate variability and change: Part I-Variability and correlation structure. Climate Dyn., 20, 491-502, doi:10.1007/ s00382-002-0286-0 .

Bretherton, C. S., C. Smith, and J. M. Wallace, 1992: An intercomparison of methods for finding coupled patterns in climate data. J. Climate, 5, 541-560, doi:10.1175/1520-0442(1992)005<0541: AIOMFF $>2.0 . \mathrm{CO} ; 2$.

Broccoli, A. J., N.-C. Lau, and M. J. Nath, 1998: The cold oceanwarm land pattern: Model simulation and relevance to climate change detection. J. Climate, 11, 2743-2763, doi:10.1175/ 1520-0442(1998)011<2743:TCOWLP $>2.0$. CO;2.

Compo, G. P., and P. D. Sardeshmukh, 2010: Removing ENSOrelated variations from the climate record. J. Climate, 23, 1957-1978, doi:10.1175/2009JCLI2735.1.

— Project. Quart. J. Roy. Meteor. Soc., 137, 1-28, doi:10.1002/ qj. 776 .

Davis, R. E., 1976: Predictability of sea surface temperature and sea level pressure anomalies over the North Pacific Ocean. J. Phys. Oceanogr., 6, 249-266, doi:10.1175/1520-0485(1976)006<0249: POSSTA $>2.0 . \mathrm{CO} ; 2$.

Deser, C., R. Tomas, M. Alexander, and D. Lawrence, 2010: The seasonal atmospheric response to projected Arctic sea ice loss in the late twenty-first century. J. Climate, 23, 333-351, doi:10.1175/ 2009JCLI3053.1.

, R. Knutti, S. Solomon, and A. S. Phillips, 2012a: Communication of the role of natural variability in future North American climate. Nat. Climate Change, 2, 775-779, doi:10.1038/ nclimate1562.

— A. S. Phillips, V. Bourdette, and H. Teng, 2012b: Uncertainty in climate change projections: The role of internal variability. Climate Dyn., 38, 527-546, doi:10.1007/s00382-010-0977-x.

,-- M. A. Alexander, and B. V. Smoliak, 2014: Projecting North American climate over the next 50 years: Uncertainty due to internal variability. J. Climate, 27, 2271-2296, doi:10.1175/ JCLI-D-13-00451.1.

Foster, G., and S. Rahmstorf, 2011: Global temperature evolution 1979-2010. Environ. Res. Lett., 6, 044022, doi:10.1088/ 1748-9326/6/4/044022.

Fyfe, J. C., N. P. Gillett, and D. W. J. Thompson, 2010: Comparing variability and trends in observed and modelled global-mean surface temperature. Geophys. Res. Lett., 37, L16802, doi:10.1029/2010GL044255.

Gillett, N. P., F. W. Zwiers, A. J. Weaver, G. C. Hegerl, M. R. Allen, and P. A. Stott, 2002: Detecting anthropogenic influence with a multi-model ensemble. Geophys. Res. Lett., 29, 1970, doi:10.1029/2002GL015836.

Hegerl, G. C., and F. W. Zwiers, 2011: Use of models in detection and attribution of climate change. Wiley Interdiscip. Rev.: Climate Change, 2, 570-591, doi:10.1002/wcc.121.

—_, and Coauthors, 2007: Understanding and attributing climate change. Climate Change 2007: The Physical Science Basis, S. Solomon et al., Eds., Cambridge University Press, 663-745.
Hurrell, J. W., 1996: Influence of variations in extratropical wintertime teleconnections on Northern Hemisphere temperature. Geophys. Res. Lett., 23, 665-668, doi:10.1029/96GL00459.

Jain, S., U. Lall, and M. E. Mann, 1999: Seasonality and interannual variations of Northern Hemisphere temperature: Equator-to-pole gradient and ocean-land contrast. J. Climate, 12, 1086-1100, doi:10.1175/1520-0442(1999)012<1086: $\mathrm{SAIVON}>2.0 . \mathrm{CO} ; 2$.

Joshi, M. M., J. M. Gregory, M. J. Webb, D. M. H. Sexton, and T. C. Johns, 2008: Mechanisms for the land/sea warming contrast exhibited by simulations of climate change. Climate Dyn., 30, 455-465, doi:10.1007/s00382-007-0306-1.

Kalela-Brundin, M., 1999: Climatic information from tree-rings of Pinus sylvestris $L$. and a reconstruction of summer temperatures back to AD 1500 in Femundsmarka, eastern Norway, using partial least squares regression (PLS) analysis. Holocene, 9, 59-77, doi:10.1191/095968399678118795.

Klein, W. H., 1962: Objective specification of monthly mean surface temperatures from 700-mb heights. J. Appl. Meteor., 1, 154-156, doi:10.1175/1520-0450(1962)001<0154:SOMMST>2.0.CO;2. , 1983: Objective specification of monthly mean surface temperature from mean $700 \mathrm{mb}$ heights in winter. Mon. Wea. Rev., 111, 674-691, doi:10.1175/1520-0493(1983)111<0674: OSOMMS $>2.0 . \mathrm{CO} ; 2$.

— ing and empirical orthogonal functions in specifying monthly surface temperature from $700 \mathrm{mb}$ data. Mon. Wea. Rev., 111, 669-673, doi:10.1175/1520-0493(1983)111<0669: ACOPSA $>2.0 . \mathrm{CO} ; 2$.

_ B. M. Lewis, and I. Enger, 1959: Objective prediction of fiveday mean temperatures during winter. J. Meteor., 16, 672-682, doi:10.1175/1520-0469(1959)016<0672:OPOFDM >2.0.CO;2.

Lawrimore, J. H., M. J. Menne, B. E. Gleason, C. N. Williams, D. B. Wuertz, R. S. Vose, and J. Rennie, 2011: An overview of the Global Historical Climatology Network monthly mean temperature data set, version 3. J. Geophys. Res., 116, D19121, doi:10.1029/2011JD016187.

Mantua, N. J., S. R. Hare, Y. Zhang, J. M. Wallace, and R. C. Francis, 1997: A Pacific interdecadal climate oscillation with implications on salmon production. Bull. Amer. Meteor. Soc., 78, 1069-1079, doi:10.1175/1520-0477(1997)078<1069: APICOW $>2.0 . \mathrm{CO} ; 2$.

McIntosh, P., A. Ash, and M. Smith, 2005: From oceans to farms: The value of a novel statistical climate forecast for agricultural management. J. Climate, 18, 4287-4302, doi:10.1175/JCLI3515.1.

Meehl, G. A., and Coauthors, 2007a: Global climate projections. Climate Change 2007: The Physical Science Basis, S. Solomon et al., Eds., Cambridge University Press, 747-845.

—, C. Covey, K. E. Taylor, T. Delworth, R. J. Stouffer, M. Latif, B. McAvaney, and J. F. B. Mitchell, 2007b: The WCRP CMIP3 multimodel dataset: A new era in climate change research. Bull. Amer. Meteor. Soc., 88, 1383-1394, doi:10.1175/BAMS-88-9-1383.

Nitta, T., and S. Yamada, 1989: Recent warming of tropical sea surface temperature and its relationship to the Northern Hemisphere circulation. J. Meteor. Soc. Japan, 67, 375-383.

Penland, C., and L. Matrosava, 2006: Studies of El Niño and interdecadal variability in tropical sea surface temperatures using a nonnormal filter. J. Climate, 19, 5796-5815, doi:10.1175/ JCLI3951.1.

Quadrelli, R., and J. M. Wallace, 2004: A simplified linear framework for interpreting patterns of Northern Hemisphere wintertime climate variability. J. Climate, 17, 3728-3744, doi:10.1175/ 1520-0442(2004)017<3728:ASLFFI>2.0.CO;2. 
Quinn, W. H., and V. T. Neal, 1984: Recent climate change and the 1982-83 E1 Niño. Proc. Eighth Annual Climate Diagnostic Workshop, Downsville, ON, Canada, NOAA, 148-154.

Santer, B. D., K. E. Taylor, T. M. L. Wigley, J. E. Penner, P. D. Jones, and U. Cubasch, 1995: Towards the detection and attribution of an anthropogenic effect on climate. Climate Dyn., 12, 77-100, doi:10.1007/BF00223722.

, and Coauthors, 2001: Accounting for the effects of volcanoes and ENSO in comparisons of modeled and observed temperature trends. J. Geophys. Res., 106, $28033-28059$, doi:10.1029/ 2000JD000189.

Smith, T. M., R. W. Reynolds, T. C. Peterson, and J. Lawrimore, 2008: Improvements to NOAA's historical merged landocean surface temperature analysis (1880-2006). J. Climate, 21, 2283-2296, doi:10.1175/2007JCLI2100.1.

Smoliak, B. V., 2009: A Eurasian pattern of Northern Hemisphere wintertime sea-level pressure variability. M.S. thesis, University of Washington, $116 \mathrm{pp}$.

- 2013: Detection and attribution of global surface air temperature change in the instrumental record. Ph.D. thesis, University of Washington, $190 \mathrm{pp}$.

_- J. M. Wallace, M. T. Stoelinga, and T. P. Mitchell, 2010: Application of partial least squares regression to the diagnosis of year-to-year variations in Pacific Northwest snowpack and Atlantic hurricanes. Geophys. Res. Lett., 37, L03801, doi:10.1029/ 2009GL041478.

Sutton, R. T., B. Dong, and J. M. Gregory, 2007: Land/sea warming ratio in response to climate change: IPCC AR4 model results and comparison with observations. Geophys. Res. Lett., 34, L02701, doi:10.1029/2006GL028164.

Thompson, D. W. J., J. M. Wallace, and G. C. Hegerl, 2000: Annular modes in the extratropical circulation. Part II: Trends. J. Climate, 13, 1018-1036, doi:10.1175/1520-0442(2000)013<1018: AMITEC $>2.0 . \mathrm{CO} ; 2$.

- — - P. D. Jones, and J. J. Kennedy, 2009: Identifying signatures of natural climate variability in time series of global-mean surface temperature: Methodology and insights. J. Climate, 22, 6120-6140, doi:10.1175/2009JCLI3089.1.

Van den Dool, H. M., E. A. O'Lenic, and W. H. Klein, 1993: Consistency check for trends in surface temperature and upper-level circulation: 1950-1992. J. Climate, 6, 2288-2297, doi:10.1175/1520-0442(1993)006<2288:CCFTIS >2.0.CO;2.

Vose, R. S., and Coauthors, 2012: NOAA's merged land-ocean surface temperature analysis. Bull. Amer. Meteor. Soc., 93, 1677-1685, doi:10.1175/BAMS-D-11-00241.1.

Walker, G., and E. Bliss, 1932: World Weather V. Mem. Roy. Meteor. Soc., 4, 53-84.

_ , and — 1937: World Weather VI. Mem. Roy. Meteor. Soc., 4, 119-139.

Wallace, J. M., 1995: Natural and forced variability in the climate record. Natural Climate Variability on Decade-toCentury Time Scales, D. G. Martinson et al., Eds., National Academy Press, 199-210.

_ C. Smith, and C. S. Bretherton, 1992: Singular value decomposition and wintertime sea surface temperature and 500-mb height anomalies. J. Climate, 5, 561-576, doi:10.1175/ 1520-0442(1992)005<0561:SVDOWS >2.0.CO;2.

—, Y. Zhang, and J. A. Renwick, 1995: Dynamic contribution to hemispheric mean temperature trends. Science, 270, 780-782, doi:10.1126/science.270.5237.780.

— - , and L. Bajuk, 1996: Interpretation of interdecadal trends in Northern Hemisphere surface air temperature. J. Climate, 9, 249-259, doi:10.1175/1520-0442(1996)009<0249: IOITIN $>2.0 . \mathrm{CO} ; 2$.

, Q. Fu, B. V. Smoliak, P. Lin, and C. M. Johanson, 2012: Simulated versus observed patterns of warming over the extratropical Northern Hemisphere continents during the cold season. Proc. Natl. Acad. Sci. USA, 109, 14337-14342, doi:10.1073/ pnas.1204875109.

Zhang, Y., J. M. Wallace, and D. S. Battisti, 1997: ENSO-like interdecadal variability: 1900-93. J. Climate, 10, 1004-1020, doi:10.1175/1520-0442(1997)010<1004:ELIV>2.0.CO;2. 